\title{
Las ciencias en la educación primaria
}

\author{
Javier Fuster
}

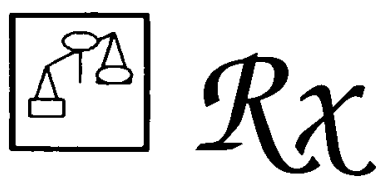

La enseñanza de los contenidos cientificos ha estado tradicionalmente reservada a las etapas de la enseñanza secundaria cuando se suponía que los alumnos estaban capacitados para la formalización del pensamiento abstracto. Los autores de este trabajo propugnan iniciar el camino del aprendizaje científico desde la educación primaria a partir de una secuenciación basada en el desarrollo de los conceptos en edades tempranas.

\section{INTRODUCCION}

La producción didáctica sobre las ciencias de la naturaleza en nuestro país se ha centrado fundamentalmente en las etapas de la educación secundaria obligatoria y bachilleratos.

Una tradición iniciada a finales de la decada de los setenta ha generado, desde entonces, profusión de trabajos útiles para cualquier profesor de ésta área inquieto por su desarrollo profesional.

Las condiciones laborales del profesorado de enseñanzas medias, y seguramente una mayor proximidad a la instancia investigadora por excelencia - -aunque no precisamente en didácticas específicas-, la universidad, ha supuesto una acumulación de información en forma de tesis doctorales, artículos en revistas profesionales, bibliografia...

Precisamente muchos de estos trabajos avalaron muchas de las decisiones en las que se ha basado el desarrollo del área de Ciencias de la Naturaleza en el nuevo sistema educativo.

En cambio, la reflexión sobre el proceso de enseñanza y aprendizaje de las ciencias en las edades correspondientes a la educación primaria continua siendo un terreno bastante virgen.

Otro factor además de los señalados anteriormente ha podido influir: la propia concepción de la ciencia y en último término, el nivel de inteligibilidad al que se está dispuesto a llegar sin que la ciencia pierda su sentido.

La educación primaria corresponde al tramo comprendido entre los seis y los doce años de edad. ¿Se puede enfrentar a estos escolares con cuerpos teóricos de conocimiento, por ejemplo, las leyes de Newton? Pues, seguramente no, vistos los resultados que se obtienen cuando se intenta enfrentarlos a edades superiores. ¿Són las teorias de Newton la entrada al mundo de la ciencia? Podriamos continuar y logra- 
riamos una serie infinita de preguntas, pero en cualquier caso no parece el camino más certero. Los niños y las niñas en nuestro país estan escolarizados obligatoriamente desde los seis años. Puede ser mucho más relevante plantearnos: ¿Qué enseñarles?, ¿ cómo enseñarlo? y ¿para qué y por qué enseñarlo?. Reflexionar sobre si lo que enseñamos es ciencia, preciencia o paraciencia, según ocasiones, no són más que obstáculos que perpetuan la falta de curricula científicos en nuestro pais para estas edades.

Son muchas las cuestiones, curiosidades e inquietudes que se plantean nuestros niños y nuestras niñas a las que la ciencia a lo largo de su historia ha dado respuestas y que a través de un esfuerzo didáctico se puede conseguir que inicien el camino hacia ellas, dandole sentido a la propia etapa.

Parece indudable que una buena planificación de la enseñanza a lo largo de su sistema educativo obligatorio, deba aspirar a rentabilizar cada uno de sus tramos de manera que como resultado de ella, se cumpla el objetivo de que cada niño y niña entiendan este mundo para poder actuar en él.

De esta forma, la educación primaria debe ser el momento en el que se inicie el recorrido, en el que de una forma intencionada y sistemática se impulse el cambio de teoria desde las representaciones de los niños hacia los contenidos científicos establecidos. Y sobre todo el momento en el que se inice el cambio respecto de cómo se forman los conceptos y por tanto de cómo se piensa sobre ellos.

Esta etapa, es el espacio en el que debe comenzar la manipulación de objetos, instrumentosy aparatos, la experimentación de hipótesis, el surgimiento del pensamiento como tentativa de explicación del mundo natural, la planificación de investigaciones destinadas a validar o refutar una idea, etc.

Aproximarlos a la imagen de la ciencia y al que significa hacerla debe ser el contenido y el marco por excelencia en el que se desarrolle la etapa.

\section{UN ENFOQUE GLOBALIZADOR}

Desde esta perspectiva, un equipo de seis profesores especializados en diferentes áreas del currículo de la educación primaria, nos pusimos a trabajar hace cuatro años en la elaboración de propuestas para cada uno de los ciclos que tuviesen un fuerte substrato científico. Mi afinidad, dada mi formación en ciencias y la constatación de la ausencia de propuestas con este enfoque fueron el origen de la elaboración de un proyecto para la etapa completa, a punto de finalizarse en estos momentos.

Inicialmente, la inexistencia de un área diferenciada de ciencias en la etapa, como si existe en otros paises, pudo parecer un obstáculo. De hecho, creemos que su no aparición en la relación nominal de las áreas de la educación primaria ha podido disuadir a más de un «experto».

Esta ausencia evidente ha originado que de momento seamos profesores de esta etapa los que hemos empezado la andadura. En cualquier caso, creemos que efectivamente nos corresponde en primer término decidir cuales son las ciencias de la etapa. Aunque no en solitario. Hemos trabajado de forma estrecha con especialistas de esta área en educación secundaria, lo que nos ha supuesto un refrendo sobre la cientificidad de nuestras propuestas y sobre todo, nos ha permitido realizar un diseño que no supone saltos en el vacio cuando se produce la transición entre las dos etapas.

El hecho de que los contenidos de ciencias estén recogidas en el área de Conocimiento del Medio Natural, Social y Cultural induce a planteamientos globalizadores, lo que conlleva la ventaja de que los problemas que se plantean para hacer avanzar el conocimiento son mucho más próximos a los que mueven la curiosidad del escolar, estando por tanto fuertemente contextualizados en su vida cotidiana. 
De esta forma, las disciplinas no son un punto de partida, sino un punto de llegada, el cual se alcanza para organizar el conocimiento que se va construyendo al conferirle significados capaces de explicar la realidad.

Otro de los elementos fundamentales en esta perspectiva globalizadora al diseñar las actividades de enseñanza de las ciencias ha sido el trabajo continuo e integrador con el área de Lenguas. Las ciencias dan el contexto en el que se pueden desarrollar las competencias comunicativas: hablar, escribir, leer, escuchar. Sobre las rocas y los minerales, los circuitos eléctricos, la reflexión de la luz, etc se pueden realizar descripciones, listados, monografías, encuestas, audiciones, lecturas informativas...

De la misma manera, las Matemáticas, la Educación Artística y el resto de las áreas aprovechan el contexto creado por las ciencias aunque sin forzar nunca su presencia. Aquellos contenidos que en un tratamiento coherente e independientemente del área de procedencia contribuyan al desarrollo de un tópico, se insertan. Los que no puedan trabajarse desde una perspectiva globalizadora, se trabajan independientemente en el área correspondiente.

Este planteamiento integrador nos ha permitido adecuar propuestas horarias más allá de los típicos cuadros de horas/áreas, lo que ha supuesto una máyor flexibilidad y que las producciones de los discentes mejoren significativamente, al ser objeto de aprendizaje en el mismo ámbito, los contenidos científicos, la comprensión de textos, la producción de los mismos, etc.

\section{PRINCIPIOS DE PROCEDIMIENTO}

El aprendizaje por investigación como propuesta metodológica supone que el escolar trabaja en contextos de investigación en el que adiestra tanto en la planificación y ejecución de investigaciones completas como en la resolución de cada una de sus partes. Por tanto, delimitará problemas, emitirá hipótesis, seleccionará materiales y procesos para contrastar sus ideas, registrará resultados, comunicará conclusiones, etc.

Según la entidad de la investigación convendrá que se ejercite en uno u otro de los elementos citados, sin pretender llevar a cabo ningún tipo de ordenación.

Se puede pasar de una investigación en la que los escolares deban elegir entre varias hipótesis ya dadas para resolver un problema determinado, a otra en la que únicamente conocen el tema y deben definir problemas interesantes y susceptibles de ser investigados en la escuela, a continuación de otra en la que conocen el problema, la hipótesis, los materiales necesarios, el proceso que deben seguir y únicamente se trata de resolver el tipo de registro y el procedimiento para comunicar sus resultados.

Lo que si debe ser objeto de secuenciación es la expectativa con respecto a su capacidad de resolución. Al principio, cuando se plantea una investigación la pregunta del profesor es del tipo «Dime que vas a hacer». En cambio, cuando ya tienen experiencia, coincidiendo normalmente con el final de la etapa, el diseño de las investigaciones debe explicitar el juego de las variables (independiente, dependiente y controlables)

Si cualquier niña aficionada a la pesca es capaz de discernir los factores de los que depende una buena captura, puede igualmente decidir cual es la variable manipulable o independiente, cual depende de ella y cuales debe tener controladas, si lo que se trata de demostrar es la hipótesis: «A mayor riego, mayor crecimiento de una planta».

Trabajar en contextos de investigación en estas edades supone tener en cuenta que:

Poseen una «teoría del mundo», es decir, cuando se enfrentan a un problema poseen unas ideas previas que condicionarán en primer término su investigación. 
Los niños y las niñas, como cualquier persona a lo largo de su vida, van adquiriendo un conjunto de ideas, que les permiten encontrar sentido a sus acciones, como por ejemplo la necesidad de comer, o los sucesos que ocurren en su entorno, como puede ser la caída de una manzana de un árbol. Este conjunto de ideas son consustanciales a las personas y gracias a ellas no vivimos en estado de desorientación permanente.

Las ideas, sean erróneas o verdaderas, surgen de una manera natural, sin poder relacionarlas directamente con ninguna enseñanza específica, a no ser el mismo hecho de vivir. Un porcentaje muy elevado de las situaciones cotidianas a las que se enfrentan los escolares puede ser explicada con este conjunto de ideas espontáneas.

El conocer las ideas de partida que poseen los escolares es imprescindible para poder conectar con la visión del mundo que poseen y así poder transformarlo, acercándola a una visión más científica de la realidad.

Aunque las características de estas ideas coinciden en muchos casos con las expuestas en muchos trabajos de investigación como, por ejemplo, los descritos por Driver, R.; Guesne, E. y Tiberghien, A. (1985) me gustaría llamar la atención sobre algunas peculiaridades propias de esta etapa, en la qué además de la inexperiencia respecto de los contenidos científicos se presenta una ausencia de otras competencias.

- La imposibilidad de verbalizarlas no implica que no existan. Un escolar que respecto a cualquier cuestión, responda con un «no lo sé», no debe entenderse como que no tiene ideas al respecto. Seguramente lo que falla es la técnica o el ambiente para que puedan expresarlas sin ningún tipo de vergüenza o temor a la sanción. Tener en cuenta esta característica es fundamental en la etapa primaria. Utilizar diferentes técnicas ( ayudas en la verbalización, realización de procesos, utilización del lenguaje plástico, ambiente afectivo y distendido, etc. ) es necesario para poder conectar con ellas. Así mismo, resulta fundamental que el profesor no actue de una forma sancionadora ni positiva ni negativamente y que además actue para que el ambiente tampoco lo sea.

- Las ideas previas tienen un grado de abstracción limitado, especialmente en esta etapa, ya que proceden de experiencias concretas y resultan por tanto alejadas de los conceptos científicos, y por tanto son básicamente incorrectas, aunque obviamente los niños adquieren espontaneamente muchos conceptos que son correctos o están en la via de serlos

- Las ideas previas son prácticamente ubicuas, es decir, los niños y las niñas tienen ideas, normalmente, de todas las cosas, por lo que se debe considerar que nuestros escolares poseen ideas previas al acto docente de todos los contenidos curriculares.

Una vez vistas estas características propias de las ideas previas en esta etapa es necesario tener en cuenta que al conocerlas, únicamente vemos la punta del iceberg, pero que esta zona es la única a la que podemos agarrarnos para poder caminar con ellos desde su visión del mundo hacia otra más cercana a la de la ciencia.

\section{EL LARGO Y TORTUOSO CAMINO DE LA FORMACION DEL CONCEPTO}

A través del proceso de enseñanza y aprendizaje los docentes favorecemos la elaboración de nuevos conocimientos que se incorporan a los esquemas mentales de nuestros escolares.

La entrada de nueva información interacciona con su visión de la realidad, produciendo una remodelación de ésta, siempre que le resulte significativa, es decir, siempre que conecte con sus ideas y le proporcione otras más explicativas que las que poseía. 
Hay que tener en cuenta que ante cualquier situación nueva, se produce una intuición ( no basada en el razonamiento ) de por donde puede ir, sin entenderlo completamente. A partir de aquí se construye, mediante un esfuerzo para confirmar la intuición, por medio del razonamiento lógico.

Desde este punto de vista, más que extraer información de la realidad, se produce una construcción de ésta de forma dinámica: el concepto no queda fijado, sino que se hace servir, se amplia, se duda sobre la idea previa y sobre la nueva, se sustituye, se prueba, se vuelve a la intuición inicial... Si al final de todo el largo proceso existe acuerdo, el nuevo concepto se incorpora, rechazándose como falto de sentido en caso contrario.

Cabe no olvidar por otra parte, que los conceptos no se construyen en forma de «todo o nada», sino que se intuyen vaga y oscuramente, ganando amplitud, profundidad y claridad con la maduración y la experiencia. Por ello, es importante establecer estrategias aproximativas y no pretender llegar directamente al concepto definitivo, sobre todo en esta etapa y teniendo en cuenta la complejidad de la mayoría de los conceptos científicos. Por tanto, no se trata por ejemplo de plantear directamente el concepto de fuerza, sino utilizar definiciones que mejoren su idea inicial, la hagan más explicativa, y estén dirigidas hacia la definición correcta actual, aunque nos quedemos en una etapa intermedia.

Para trabajar con las ideas previas de los escolares, es necesario reconocer la existencia de una doble coherencia. Aquello que a los ojos del maestro puede ser un razonamiento claro y riguroso, puede no serlo para el escolar. De ahí la necesidad de conocer cuáles son los mecanismos intelectuales que acciona el estudiante de la etapa primaria, de manera que se puedan diseñar estrategias que permitan acompañarlo en su aprendizaje, teniendo en cuenta su ritmo evolutivo y los factores que lo condicionan: los aprendizajes anteriores, la inteligencia natural, la motivación y el medio cultural.

\section{Hacia la transformación de las ideas}

Esta visión de la construcción del conocimiento científico hace necesaria la concurrencia de los siguientes momentos didácticos:

a) El momento de la detección y diagnóstico de las ideas previas. El escolar, y el profesor, deben ser conscientes de las ideas de las que parten, para lo cual se pueden utilizar diferentes técnicas:

- entrevista abierta o estructurada sobre conceptos, procedimientos, sucesos con predicción y explicación, actitudes, etc.

- realización de procesos con predicción y explicación,

- asociación de palabras o símbolos,

- dibujos con explicación,

- observación por parte del profesor,

- cuestionarios, abiertos u objetivos.

En todas ellas, en esta etapa, es muy importante la ayuda en la verbalización.

b) Ha de generarse en el alumno la necesidad — la motivación - para que abandone sus esquemas mentales cuando no sean pertinentes, mediante el planteamiento de conflictos o contraejemplos, considerando sus errores como paso inevitable en la construcción del conocimiento científico, y en cualquier caso resaltando la parte de acierto que tienen sus ideas.

c) Se ha de aplicar una estrategia de actuación que permita una nueva conceptualización, al nivel correspondiente y de acuerdo con el saber científico. Es propiamente el punto que corresponde a la elaboración de nuevos conocimientos, una vez que se han 
hecho explícitas las ideas previas y se produce un conflicto que motive a encontrar otras ideas que funcionen mejor. Los conocimientos así adquiridos se han de poder aplicar en diferentes situaciones, tanto académicas como no académicas, para que su significado sea completo. No tenerlo en cuenta puede hacer perder el carácter de construcción de significados reales. No se trata de elaborar saberes académicos que únicamente tengan sentido dentro de la escuela y que les permita aprobar una evaluación, sino de ir mucho más allá. Se trata de incidir en la elaboración de conocimientos que les puedan servir para poder interpretar su vida en el mundo.

d) Como último punto, se ha de establecer una reflexión sobre el propio proceso de aprendizaje, que desvele tanto lo que se ha aprendido como el proceso que se ha seguido para aprenderlo, sirviendo para comparar lo que sabían y lo que saben, cuestión que les muestra el sentido del esfuerzo que han realizado motivandolos para próximos aprendizajes. Así mismo, la reflexión sobre las técnicas de trabajo que han utilizado, pueden ir mostrándoles la eficacia y las dificultades que para cada uno tienen. No hay que olvidar todo el aspecto afectivo con respecto al mismo proceso (me he aburrido, me ha gustado, mi grupo no ha funcionado bien...), que proporciona información sobre el ambiente de aprendizaje y la interacción en el aula.

Los puntos presentados no deben entenderse como una secuenciación temporal, sino como un conjunto de estrategias que se ponen en juego continuamente. No siempre se empieza con unas ideas previas, se continua con un conflicto, después viene la construcción y al final la reflexión. Durante el proceso, continuamente saldrán ideas, que harán construir otras nuevas, que plantearán nuevos conflictos, etc.

\section{APRENDER TRABAJANDO EN UN CONTEXTO DE INVESTIGACION}

La relación de momentos didácticos que hemos visto, caracteriza el trabajo en el aula como una actividad investigadora, tanto para el alumnado como para el profesorado. Éste, además de su tarea como investigador didáctico, también lo es con respecto a los contenidos científicos. Presentarse como tal en el aula, favorece un tipo de relaciones mucho más afectiva al reconocer las limitaciones, la ignorancia y los errores que todos tenemos, sin menoscabo de la diferencia específica dada por la experiencia y el saber. Esta situación, además de mostrar de una manera mucho más clara la realidad, permite al escolar relativizar la información.

La formación de conocimientos adquiere su verdadera dimensión didáctica cuando se organiza el aprendizaje mediante la propuesta y resolución de problemas. Estos favorecen el establecimiento de relaciones entre los hechos y los conceptos y plantean la necesidad de utilizar procedimientos específicos en los cuales los alumnos irán adiestrándose a medida que su investigación lo requiera, asentando al mismo tiempo, el desarrollo de actitudes. Por tanto, conceptos, procedimientos y actitudes han de considerarse integrados en una metodología general y no como elementos independientes con entidad suficiente para generar aprendizaje.

Se trata de proporcionar oportunidades para que los escolares de la etapa primaria a partir de percepciones y sensaciones, puedan cuestionarse la realidad y, mediante la refléxión personal y la interacción social, elaborar respuestas a los interrogantes del mundo. De esta forma, a partir del pensamiento espontáneo, del lenguaje y de la nueva información irán construyendo esquemas de conocimiento, en un proceso de aproximación sucesiva hacia las ideas establecidas científicamente, 
produciendo cada vez explicaciones más coherentes y de un grado de racionalidad mayor.

$Y$ de establecer condiciones para que generen ideas y aprendan a pensar en contextos y con argumentos quie tengan sentido para los niños y las niñas.

Precisamente una de las posibilidades más interesantes de la actividad investigadora es el cuestionamiento de sus propias ideas, lo que permite un tratamiento individualizado de las representaciones personales de los alumnos, dando lugar a que frente a un mismo problema aparezcan diferentes hipótesis (respuestas ), con diferentes procedimientos para contrastarlas, potenciando en el aula la presencia del pensamiento divergente, que bajo una óptica de respeto e interés permite ofrecer distintas visiones que pueden complementarse o reformular las premisas iniciales, mejorándolas.

Los problemas, en cualquier caso, deben dirigirse a construir experiencias acerca de cómo son y cómo funcionan las cosas, al mismo tiempo que sirven para aprender a usar el lenguaje para dar significado a las «reglas» elementales que subyacen en el desarrollo de los diferentes fenómenos.

Se trata de entender el proceso de enseñanza y aprendizaje, como el desarrollo de formas de mirar cognoscitivamente la realidad, de operar sobre ella concretamente, de reflexionar y de entender en sentido general la conexión entre experiencia, lenguaje y conocimiento.

En resumen, establecer el proceso de enseñanza en torno al aprender trabajando, de forma que sea la propia necesidad de responder a preguntas o elaborar proyectos, lo que suscite la búsqueda de nuevas informaciones, la verificación de las mismas, la discriminación por su relevancia, el establecimiento de relaciones, el uso de modelos explicativos y la comunicación de las respuestas halladas o de los proyectos realizados.

El proceso de enseñanza y aprendizaje, vertebrado de esta manera, hace necesario plantearse la selección de los contenidos, de manera que se opte por un estudio profundo de los que se consideren más relevantes, frente a la visión superficial de una gran cantidad de ellos.

\section{SILENCIO, SONIDO: MUSICA}

Por ejemplo, una de las unidades de este proyecto para el primer ciclo de la etapa que desarrolla un tratamiento globalizado con contenidos de música es la que tiene como título el de este apartado.

Respecto a las propuestas de ciencias, se les hace «oir» con un nuevo oido el sonido y sus caraterísticas.

Se les plantea inicialmente la determinación del órgano gracias al cual se produce la audición y los cuidados que requiere para su buen funcionamiento, Se trata de propiciar que hablen de sus experiencias, de lo que hacen y deben hacer, de las enfermedades que han padecido o conocen del oido, de las situaciones o acciones que pueden ser peligrosas. Este conjunto de actividades finaliza registrando consejos para el buen funcionamiento del oido.

Posteriormente, se les plantea las similitudes y diferencias entre las orejas de todad la clase. ¿Existen dos de vosotros que tengais las orejas iguales?. Por parejas, observan las orejas y las dibujan, formando con todos los dibujos un "panel orejudo». Después se trata de observar parecidos y sobre todo de constatar que no hay dos escolares que tengan las orejas iguales. Puede servirles para entender mejor la riqueza de la diversidad. 
¿Por qué tenemos dos orejas?, es un problema que se les plantea para que ellos digan sus ideas, las discutan y esten dispuestos a comprobarlas. ¿Cómo hareis para demostrarlo? ¿Qué material necesitais?. El profesor ofrece pistas cuando se producen bloqueos. Necesitais algo que no suene muy fuerte. ¿Cómo podeis anular la vista?. La prueba que hagais debe permitir comparar el sonido con las dos orejas del mismo sonido con una sola oreja. ¿Cómo conseguir que una oreja no oiga?

La realización de esta experiencia les permite conocer que el hecho de disponer de dos orejas nos permite localizar los sonidos en el espacio.

Otra actividad es grabar su voz y ver si la reconocen entre muchas. ¿Por qué nos resulta extraña nuestra propia voz? Observad cual es el camino por el que nos llega a nuestro oido y comparadlo con el que sigue cuando lo oimos a través de una grabación.

El diapasón es un aparato que permite realizar multitud de juegos y actividades. Se les invita a hacerlo sonar sobre diferentes objetos, comprobando las diferentes resonancias, previendo en ocasiones lo que puede suceder antes de colocarlo. Después de percutirlo se introduce en una cubeta y se ven las ondas que se forman, sugiriendose la explicación de que en el aire, aunque no lo veamos, también ocurre así la transmisión del sonido.

Construyen un detector de vibraciones, con un tubo de cartón, un pedazo de globo inchable y un pequeño espejo pegado sobre la membrana bien tirante. Al hablar fuerte por el extremo vacio y reflejar la luz sobre el espejo se percibe perfectamente la vibración, origen de cualquier sonido.

La construcción del detector se les plantea con un texto informativo según un modelo de proceso con el que tienen que poner en juego su capacidad comprensiva para poder realizarlo. De esta forma y muchas otras se incardina el trabajo con los contenidos del área de Lenguas.

El paisaje sonoro y la discriminación de sonidos son otro conjunto de actividades que se realizan. La audición no siempre recibe la atención ni la educación que requiere. Adivinar de quien es la voz que se escucha, que objeto se ha caido, que instrumento suena, que se oye en el exterior del colegio, o en la clase de al lado, permite descubrir diferentes timbres sonoros y acostumbrarlos a la discriminación auditiva.

Esta necesidad permite plantear reflexiones y debates sobre la importancia del silencio en la clase, ya que les permite descubrir y recrearse en un mundo sonoro que de otra forma pasa desapercibido, además de los beneficios que supone para la tranquilidad y la concentración de las personas que conviven en el aula. Las decisiones de la asamblea de aula que se realice con este motivo se recogen en forma de acuerdos.

La creación de un ambiente silencioso permite prestar oidos nuevos a las situaciones que habitualmente se producen en la clase: echar algo en la papelera, borrar la pizarra, intervenir en la asamblea, andar por la clase, cerrar una puerta, etc. Todas estas acciones es posible realizarlas con mucho menos ruido cuando se atiende a la posibilidad de "oir el silencio».

Si se consigue una campana de vacio, se confecciona un lista de objetos autónomos sonoros para comprobar que no percibimos su sonido al extraer el aire. La imagen del despertador tradicional viendo percutir la campana sin oirla es muy reveladora.

Como en el agua si que han visto que se forman las ondas, se les plantea que hablen de los viajes del sonido en su interior, de qué animales acuáticos conocen que se comuniquen con sonidos, y de cómo comprobarian que en el agua efectivamente se produce un viaje y transmisión del sonido.

Durante todo el tiempo que se está trabajando la propuesta el profesor interviene para que verbalicen sus 'realizaciones, expresen sus ideas, hagan sus preguntas, compongan sus textos con las conclusiones, dibujen sus experimentos e investigaciones, fotografien sus montajes, etc. 
Al final todas estas producciones serviran para recordar que han aprendido y como lo han hecho.

Es necesario propiciar que en el aula se hable de cómo lo han pasado, que les ha gustado, que les ha resultado difícil, en resumen de todos aquellos aspectos vivenciales y afectivos, de cuyo conocimiento nos podemos servir para programar futuras intervenciones y de cómo hacerlas para que las vivan mejor.
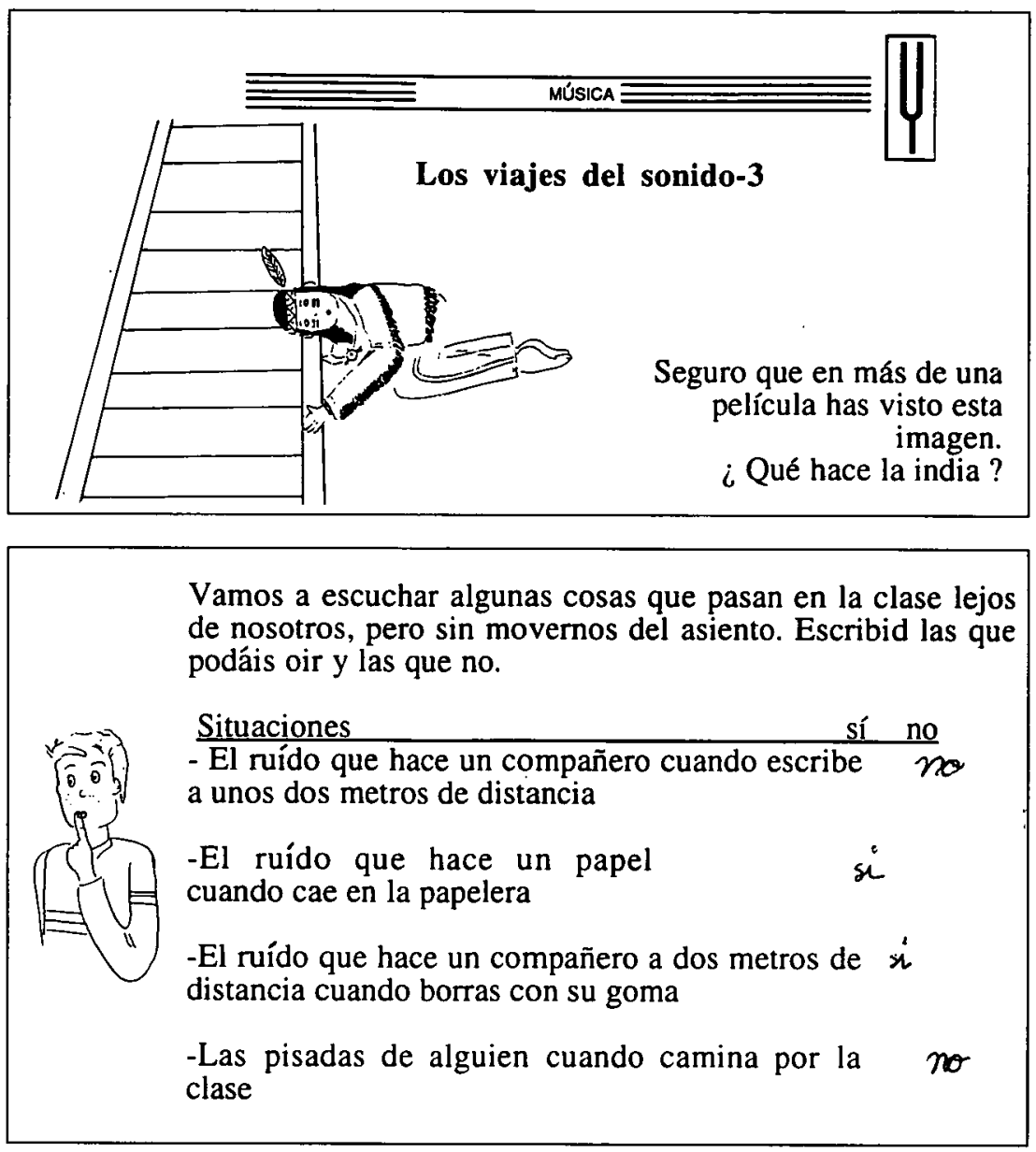

Recortes del cuaderno de trabajo de la unidad «Silencio, sonidos: música».

\section{Referencias}

ARCA, M.; MAZzolI, P., y GuIDONI, P; (1987) Guardare per sistema, guardare per variabili. Emme Edizioni. Torino

BREDERMAN, T. (1985) Laboratori programs for elementary school: A meta-analysis of efects on learning. Sci. Educación Vol 69(4)

DRIVER, R.; GUESNE, E., y TIBERGHIEN, A. (1985): Ideas cientificas en la infancia y adolescencia. Madrid: Morata /M.E.C.

GIORDAN, A. (1989) La enseñanza de las ciencias. Ed. Siglo XXI Madrid

HARLEM, W. (1989) Enseñanza y aprendizaje de las ciencias Ed. Mec-Morata. Madrid

HeMPLE, C.G. (1976) Filosofía de la ciencia natural. Ed. Alianza. Madrid

MARTIN, A., y RODRIGUEZ, A. Aproximación al proceso de emisión y verificación de hipótesis en niños de 10, 11 años. Investigación en la escuela vol. 5

TONUCCI, F. (1976) A los tres años se investiga. Ed. Avance. Barcelona. 


\section{Las ciencias en la educación primaria Javier Fuster Pérez \\ CL\&E, 1994, 22, pp. 75-84}

Resumen: A partir de la ausencia de inciativas en el campo de la didáctica de las ciencias para la etapa primaria, se explica la fundamentación de un proyecto para esta etapa elaborado y experimentado a lo largo de estos cuatro últimos años, a punto de finalizarse. Se muestran los principios metodológicos y las características de la enseñanza y aprendizaje de los contenidos científicos en las edades de 6 a 12 años, y se ejemplifican mediante la descripción de una de las propuestas de trabajo de dicho proyecto.

Datos sobre el autor: Javier Fuster Pérez. Coordinador de Educación Primaria del Servicio de Programas Curriculares. Conselleria de Educación y Ciencia de la Comunidad Valenciana.

Dirección profesional: Conselleria de Educación y Ciencia. Servicio de Programas Curriculares. Avda. de Campanar, 32. Valencia 46014. Tno. (96) 3863254.

Dirección particular: Guillem de Castro, 57-22. Valencia 46008. Tno. (96) 3517197.

(C) PERMISOS PARA CITAR O REPRODUCIR EN OTRAS FUENTES: Se pueden citar libremente hasta 500 palabras. Para reproducir una porción de texto mayor, figuras o ilustraciones, se deberá pedir permiso por escrito a la revista, especificando el uso al que se destina el texto. En todos los casos, se deberá citar el copyright de $C L \& E$. En el caso de artículos o textos que hayan sido a su vez reproducidos en $C L \& E$ los interesados deberán dirigirse tanto a los detentadores del copyright original como a $C L \& E$, en el caso de que se quiera hacer uso de la traducción. FOTOCOPIAS: Para todo lo relacionado con el uso mediante fotocopia del material de esta revista, deberán dirigirse a: CEDRO, C/ José Marañón, 10, $3 .^{\circ}$ Izda. Tel. 5941575 . Fax 4453567 